\title{
Meniscectomy: indications, procedure, outcomes, and rehabilitation
}

\author{
This article was published in the following Dove Press journal: \\ Orthopedic Research and Reviews \\ 21 December 2013 \\ Number of times this article has been viewed
}

\section{Hermann Anetzberger' \\ Christof Birkenmaier ${ }^{2}$ \\ Stephan Lorenz ${ }^{3}$ \\ 'Orthopädische Gemeinschaftspraxis am OEZ, Munich, Germany; \\ ${ }^{2}$ Department of Orthopedics, Ludwig- Maximilian-University Munich, Munich, Germany; ${ }^{3}$ Department of Orthopedic Sports Medicine, Technical University of Munich, Klinikum rechts der Isar, Munich, Germany}

Correspondence: Hermann Anetzberger Orthopädische Gemeinschaftspraxis am OEZ Hanauerstr 65, 80993 Munich, Germany

Email dr.anetzberger@orthospezial.de
Abstract: Meniscal injuries are among the most frequent reasons for knee problems. The goal of this manuscript is to review the biomechanical relevance of the human knee's menisci in relation to surgical indications, surgical techniques, rehabilitation, and outcomes. In order to identify the relevant literature, we performed a PubMed search for the years ranging from 1980-2013 using the following search terms: meniscus; biomechanical function; meniscectomy; meniscal repair; and clinical outcome. The meniscus helps to distribute the forces between the tibial and femoral articular cartilage layers in a load-sharing capacity. Meniscus damage or meniscectomy intuitively leads to an overloading of the cartilage and, hence, to the development of osteoarthrosis. Precise knowledge of meniscal shape and function, of the type of injury, of surgical techniques, as well as of postsurgical rehabilitative care are of decisive importance for an individually-adjusted treatment strategy. Other underlying coexisting knee pathologies also need to be considered. The diagnosis of a meniscal injury is based upon clinical history, physical examination, and imaging studies. The treatment of a meniscal lesion includes conservative, as well as operative, procedures. The goals of surgery are to reduce pain and disability, as well as to preserve meniscal function without causing additional cartilage damage. The resection of meniscal tissue should be restricted to as much as is necessary, and as little as is reasonably possible. Postoperative rehabilitation serves the purpose of improving functional deficits and pain, as well as of restoring a good range of motion and preventing secondary damage. It is the surgical therapy that dictates the aftercare, and with regards to the latter, there are clear differences between meniscectomy and meniscal repair.

Keywords: meniscectomy, osteoarthritis, meniscus pathology, meniscus function

\section{Introduction}

Ten percent of 60-year-olds suffer from knee osteoarthrosis (OA), and this percentage increases with age. ${ }^{1,2}$ Beyond age, sex, body mass index, and genetic predisposition, meniscal injuries are an important risk factor for the development of OA. ${ }^{3-5}$ After the partial or total resection of a meniscus, the local pressure at the cartilage surface increases due to a decrease of the contact area, entailing the risk of cartilage damage. The development of $\mathrm{OA}$ is dependent on time and is related to the amount of meniscal tissue loss. ${ }^{6,7}$ Complete meniscectomy almost inevitably leads to OA and, in turn, to a higher relative risk (132:1) for the implantation of a knee arthroplasty as compared to the normal population. ${ }^{8}$ It is the predominant advantage of arthroscopic meniscus surgery over open surgery to resect only the damaged parts of a meniscus and to preserve the functionally-relevant outer rim of the meniscus to reduce the risk of developing secondary OA. 
Recent studies have shown that it is not the surgical procedure, but rather the primary damage of the meniscus, that initiates the development of OA.9,10 The importance of the menisci for the knee joint dictates that these should be preserved after injuries. Whenever this is not feasible, any resection should be as limited as reasonably possible in order to avoid the onset of OA.

This review article, in its first part, focuses on the functions of the menisci. Following that, the indications for operative treatment, relevant surgical techniques, and postoperative rehabilitative measures are discussed. The relevant literature was searched in PubMed for the years ranging from 1980-2013 using the following search terms: meniscus; biomechanical function; meniscectomy; meniscal repair; and clinical outcome. The search was not restricted by language, and resulted in more than 3,000 articles. According to the topic of the paper, 300 of the most relevant papers were read. Finally, relevant data from 71 publications were extracted for the purpose of this review.

\section{Meniscus - form and function}

The essential function of a healthy joint is to distribute the active forces optimally in order to distribute them according to the loading capacity of the tissues. The forces, which act locally under dynamic loading situations onto the contact surface, are distributed uniformly via the cartilage-bone interface. ${ }^{11,12}$ The meniscus plays a pivotal role in load bearing and load transmission to the cartilage and the subchondral bone. Due to the different curvatures of the femoral condyles and the medial and lateral tibial plateaus, the main function of the meniscus is to enlarge the contact surface (Figure 1). In contrast to the medial meniscus, the lateral meniscus covers a larger part of the tibial articular surface (lateral

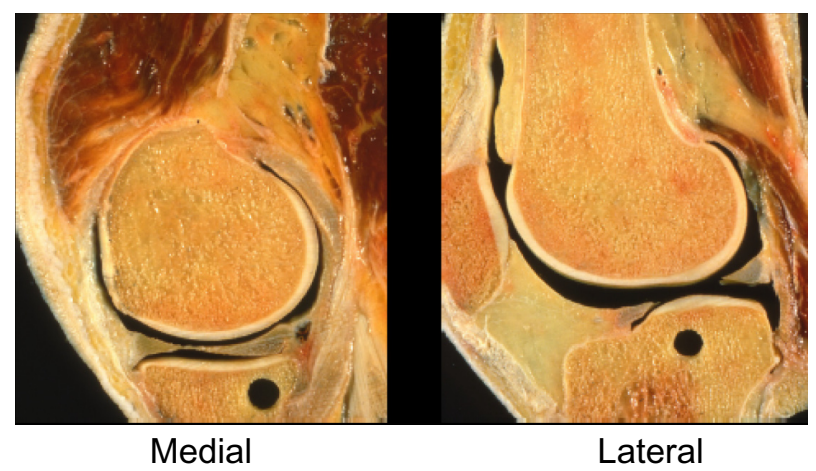

Figure I Sagittal sections through the medial and lateral tibial plateau. Notes: The curvature of the medial femoral condyle is circular in shape, whereas that of the lateral condyle is of spiral shape. The curvature of the medial tibial plateau is concave and that of the lateral tibial plateau is convex. joint surface: $75 \%-93 \%$; medial joint surface: $51 \%-74 \%){ }^{13}$ After removal of the meniscus, the contact area decreases by $40 \%$ medially, and by as much as $52 \%$ laterally. ${ }^{14}$ Another important function is that the shear forces are transformed into circular tensile stress, thus reducing the forces that act on the cartilage. ${ }^{15}$ The meniscus is stressed by radiallydirected shear forces, but because of the bony attachment at both ends, the shear forces are transformed into circular tensile stress. The arrangement of collagen fibers reflects the pattern of tensile stress trajectories. The functional significance of these marginal circular fibers is emphasized by the fact that segmental defects, complete radial tears, or root tears with complete interruption cause similar effects as a complete meniscectomy. ${ }^{16-18}$ On the other hand, a partial meniscectomy with an intact marginal ridge still has a good residual function.

The menisci also contribute to the stability of the knee joint. The loss of a meniscus leads to increased anteriorposterior laxity in the femorotibial joint, ${ }^{19}$ which becomes especially relevant in situations with an associated loss or insufficiency of the anterior cruciate ligament (ACL). Other important functions of the menisci include shock absorption, as well as the fact that they aid in the lubrication and nutrition of the articular cartilage.

The meniscal architecture reflects an optimal adaptation to its functions. Human menisci are typically crescentshaped with a wedge-like diameter. The morphology of the collagenous framework is largely responsible for the mechanical properties of the meniscus. This structure consists predominantly of type-1 collagen that typically exists in tissues that are under tensile stresses. The main portion of these collagen bundles is located in the center of the meniscus where they form thick, circularly layered bundle structures. The most central of these bundles are encased by a layer of lamellar collagen fibers. The surface of a meniscus consists of a tightly-woven network of fine collagen fibers. ${ }^{20}$ Both menisci are attached to the bone by means of collagen fibers. In the anterior area, fibers from the lateral and medial anterior insertions radiate into the ACL. ${ }^{21}$ In about $70 \%$ of cases, a transverse ligament connects the anterior horns of the two menisci. In contrast to the posterior horn of the medial meniscus, the posterior horn of the lateral meniscus is additionally attached by means of the menisco-femoral ligaments (Wrisberg and Humphrey). The presumed function of this attachment is that with rapid flexion movements, the posterior horn of the lateral meniscus is being pulled in a posterior direction, thus protecting it from impinging on the femorotibial joint, leading to injury. 


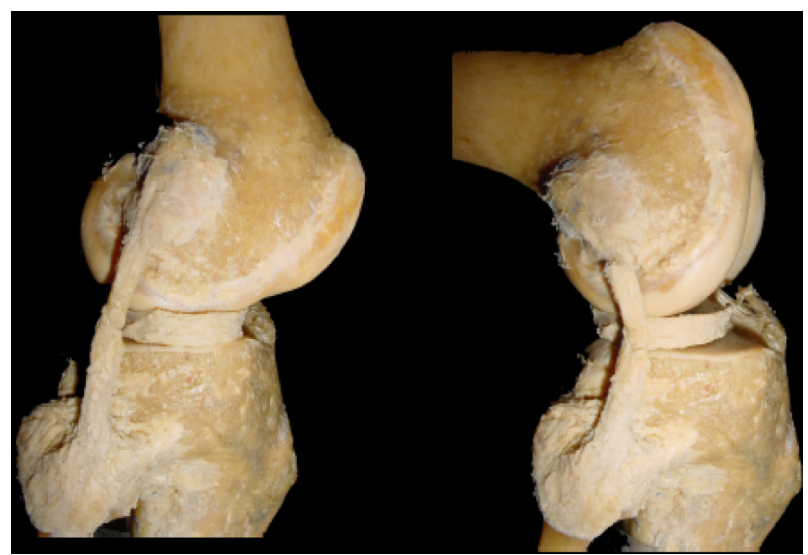

Extension

Flexion

Figure 2 Anteroposterior translation of the lateral meniscus during flexion.

The anteroposterior translation of the lateral meniscus during flexion is almost twice that of the medial meniscus (Figure 2). ${ }^{22}$ Vedi et $\mathrm{al}^{23}$ found that during the first $90^{\circ}$ of the flexion, the anterior horns undergo a dorsal excursion of $7.1 \mathrm{~mm}$ medially and $9.5 \mathrm{~mm}$ laterally, whereas the posterior horns only move by $3.9 \mathrm{~mm}$ and $5.6 \mathrm{~mm}$, respectively. Since the movement of the lateral meniscus increases even under loading conditions, range-of-motion exercises should only be performed without loading in the context of postoperative physiotherapy.

The meniscal blood supply enters from the outer rim of the meniscus with only $25 \%$ of the meniscal tissues being perfused.$^{24}$ Since good perfusion is a prerequisite for healing after a meniscal repair, the cross-section of the menisci is being divided in a red-red (outer zone), a red-white (middle zone), and a white-white (inner zone). Menisci contain nociceptors and mechanoreceptors, which are located in the outer zone, and they fulfill nociceptive as well as proprioceptive functions. ${ }^{25}$

\section{Indications for meniscus surgery}

Meniscus injuries occur either traumatically and often associated with an ACL rupture, or spontaneously as a result of structural weakening due to aging and wear. ${ }^{26,27}$ The annual incidence of meniscal injuries is approximately $66 / 100,000$ inhabitants. ${ }^{28-30}$ The portion of meniscus injuries that occur in the context of an ACL rupture is around 35\%, and about $35 \%$ of all diagnosed meniscal injuries end up being treated operatively. ${ }^{3}$

Not all meniscal injuries require surgical therapy. Tears smaller than $1 \mathrm{~cm}$, and which are stable and peripherallylocated vertical tears, as well as incomplete longitudinal tears, have a good tendency towards spontaneous healing. ${ }^{32}$
Small tears and even root tears localized at the posterior horn of the lateral meniscus also showed good long-term results with conservative treatment. ${ }^{33,34}$ The indication for surgery depends on the clinical symptoms and the degree of the injury. Other factors such as age, lower limb alignment, concomitant cartilage, and/or ligament injury, and the individual's level of activity should also play a role when deciding upon a therapeutic strategy.

The diagnosis of a meniscal injury is based on a patient's history, pain, a good physical examination, and targeted imaging. Accidents, mechanical symptoms like locking or catching, reduced range of motion, cracking, and effusion are hints that point towards a meniscal problem. The patient's history needs to be completed by asking direct questions about his or her previous injuries. Clinical tests (joint line tenderness, the Apley test, and McMurray's test) are quite reliable in diagnosing meniscal injuries. ${ }^{35-37}$ The reliability of these clinical tests is reduced with concomitant degenerative changes ${ }^{38}$ With regards to imaging studies, plain radiographs should always be performed in order to diagnose or rule out associated diagnoses such as OA, chondrocalcinosis, and rheumatoid disease. Magnetic resonance imaging (MRI) is the next logical step in imaging and, if available, it should be performed prior to deciding on a path of treatment. ${ }^{39}$ MRI can help in determining the degree and the type of a meniscal injury with very high sensitivity and specificity. ${ }^{40,41}$ Furthermore, MRI is reliable in showing associated injuries and cartilage lesions.

The most frequent indication for surgery results from the failure of a symptomatic meniscal tear to heal spontaneously, whereas a displaced or locked bucket handle tear represents an absolute indication for surgical intervention. It is important to classify meniscal injuries pre- and postoperatively as precisely as possible in order to permit the initiation of adequate, function-oriented, surgical therapy. Because of the different biomechanical functions of the medial and lateral meniscus, correct terminology regarding whether the medial or the lateral meniscus is affected is of key importance. In order to optimize the chances of success of a potential reconstructive procedure, the tear depth, tear pattern, radial location, rim width location, and the pathogenesis are of relevance beyond the shape of a meniscal tear. An overview of the classification and tear patterns is given in Table 1, as well as in Figures 3 and 4. Additional conditions, such as chondrocalcinosis, rheumatoid diseases, and the degree of OA (if existing) must be well documented, since they reduce the chances for the success of an operative procedure. 


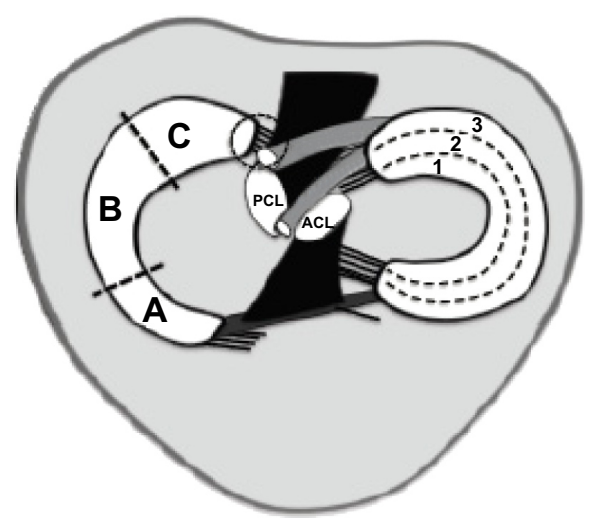

Figure 3 Zonal and regional classification of the medial and lateral meniscus. Notes: (A) Anterior, (B) mid-body, (C) posterior; I= inner zone (rim $>5 \mathrm{~mm}$; white-white), $2=$ middle zone ( $\operatorname{rim}<5 \mathrm{~mm}$; red-white), and $3=$ outer zone $(\operatorname{rim}<3 \mathrm{~mm}$; red-red).

Abbreviations: $\mathrm{PCL}$, posterior cruciate ligament; $\mathrm{ACL}$, anterior cruciate ligament.

A discoid meniscus represents a variety in the normal meniscus shape, and has a prevalence of $7 \%$ of the lateral meniscus in the normal population..$^{42}$ The therapy that is currently recommended in the case of a discoid meniscus is arthroscopic partial resection and reshaping..$^{43}$ The best results appear to be achievable when the partial resection and reshaping are completed soon after the first onset of symptoms. A root tear, which is similar to a complete radial tear, is a grave injury that leads to a complete loss of the meniscal function and therefore to the rapid development of OA; this is similar to the effects of a total meniscectomy. ${ }^{16,17}$ Whether these consequences can be averted by an operative reconstruction of the meniscal attachment remains to be seen..$^{44,45}$
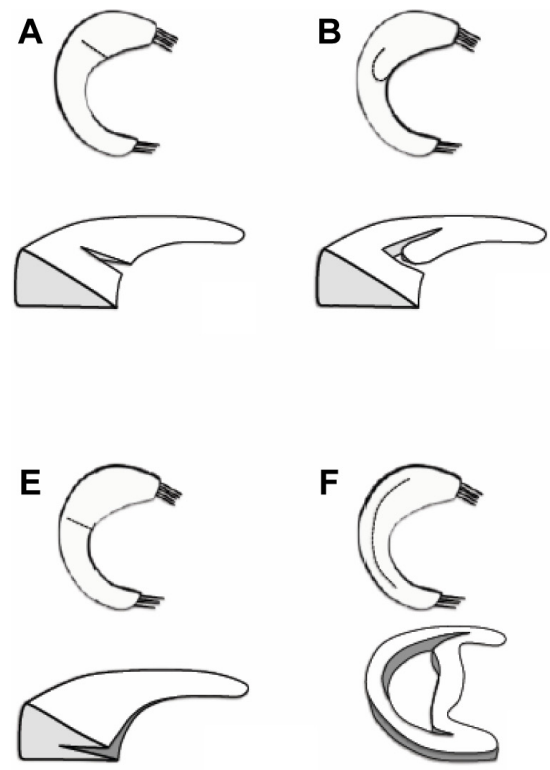

- Medial

- Lateral

- Complete

- Incomplete

- Anterior

- Mid-body

- Posterior

- Zone I (rim $<3 \mathrm{~mm}$; red-red)

- Zone 2 (rim $<5 \mathrm{~mm}$; red-white)

- Zone 3 (rim $>5$ mm; white-white)

- Longitudinal-vertical

- Horizontal

- Radial

- Vertical/horizontal flap

- Complex

- Bucket handle

- Root tear

- Traumatic

- Degenerative

In mm

- Degenerative/meniscal cyst

- Discoid meniscus

- Chondrocalcinosis

- Arthrosis

- Rheumatoid arthritis

\section{Surgical procedure}

As stated above, the paramount aim of meniscal therapy should be to maintain as much functional meniscus tissue as possible. Based on the patient's age, his or her lesion type, lesion size/depth, location (inner zone, middle zone, or outer zone), and pathogenesis (traumatic versus degenerative), the

G
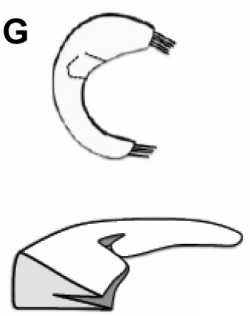

D

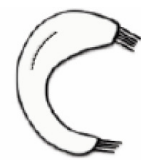

C
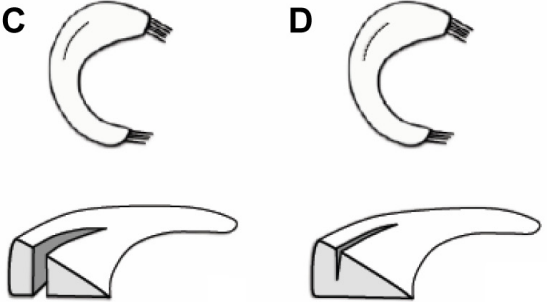

H
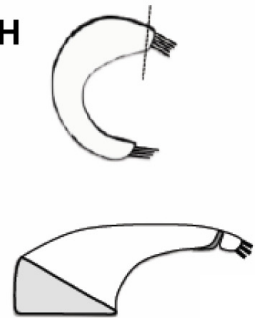

Figure 4 Meniscus tear patterns.

Notes: (A) Complete longitudinal-vertical tear; (B) incomplete longitudinal-vertical tear; (C) radial tear; (D) flap; (E) horizontal tear; (F) bucket-handle tear; (G) complex tear; and $(\mathbf{H})$ root tear. 
surgeon should be ready to perform either partial meniscectomy or meniscal suture repair. The goals of meniscectomy are to remove unstable flaps or fragments that might interfere with the femorotibial joint space by locking the joint. It also attemps to prevent lesion recurrence by repairing the inner torn portion of the meniscus, stabilizing the joint by preserving the capsular attachments of the meniscus as much as possible, and maintaining normal articular cartilage by avoiding damage during meniscectomy.

Because of their good healing potential, meniscus tears in a skeletally immature patient should be repaired regardless of the lesion's location or tear pattern. ${ }^{46,47}$ In adults, the success rate of a meniscus repair mainly depends on the lesion's location, tear pattern, and the patient's age ${ }^{47}$ To improve meniscus healing, several different techniques (such as needling, abrasion, trephination, or application of fibrin clots or platelet-rich plasma) have been recommended. ${ }^{48,49}$ Acute tears that are bigger than $1 \mathrm{~cm}$ but smaller than $4 \mathrm{~cm}$ in the red-red or red-white zone with a vertical rupture pattern in a patient younger than 40 years of age, and without axis malalignment, are good indications for meniscal repair. ${ }^{50,51}$ Furthermore, meniscus tears with a concomitant ACL rupture should be repaired, since the healing rate without treatment of the ACL is low, and given that the clinical symptoms increase over time. ${ }^{52-54}$ Conversely, because of their poor healing tendency, degenerative horizontal and complex tears in older patients and tears in the avascular zone (inner zone; Figure 3) are not recommended for meniscal repair. ${ }^{55-57}$

In our opinion, excessive resections of the medial and lateral meniscus, together with a varus or valgus malalignment of the leg, respectively, should be combined with an unloading osteotomy.

\section{Positioning and draping}

The following section describes the general procedure employed by the authors. The patient is in a supine position, and his or her knee is stabilized in a leg holder. A tourniquet is not absolutely indispensable, but it can improve visualization. The whole lower extremity is disinfected by iodine solution and the shank is covered by a stockinette. A fenestrated drape covers the contralateral leg and the rest of the body.

\section{Portal placement}

Precise portal placement is essential for arthroscopic partial meniscectomy. Since the joint space is quite narrow, lesions at the posterior horn of the menisci are often difficult to reach. If the working portal is located too proximal or too distal, the arthroscopic instruments are deflected by the femoral condyles, and excessive force is needed to reach the posterior part of the knee joint. Iatrogenic cartilage damage might result as a consequence. In contrast, deflection by the tibial head and damage of the anterior parts of the menisci might occur with portal positions that are placed too distally.

In our routine, we prefer a standard anterolateral portal (ALP) that is right underneath the tip of the inferior pole of the patella, in line with the lateral patellar border. With this portal at the "soft spot" of the knee, the risk of any cartilage damage is low, and most parts of the knee can be well visualized. Utilizing a scalpel, a vertical skin incision is made pointing towards the intercondylar notch. It should be noted that in the case of lateral meniscus lesions, the portal should be around $5 \mathrm{~mm}$ lower since the ALP might also become a working portal.

The anteromedial portal (AMP) is placed under direct visualization of the arthroscope, which is in full extension. A needle is placed right above the anterior horn of the medial meniscus. Diaphanoscopy is employed to avoid injuring venous vessels. By manipulating the needle, the accessibility of the relevant structures is checked. As a rule of thumb, the portal should be closer to the patella tendon if the intermediate part of the medial meniscus has to be addressed. For the posterior horn and the posterior root, a more medial portal is favorable. With the arthroscope pointing towards the entry point of the needle, the scalpel is advanced into the knee joint along the spinal needle. If necessary, the portal can be spread using scissors (Figure 5).

\section{Instruments}

A standard $30^{\circ}$ angulated $5.5 \mathrm{~mm}$ arthroscope is utilized in most cases. Only in very small knees (for example, in children) a smaller arthroscope is favorable. For the resection of the meniscal lesion, straight and lateral punch forceps are needed (Figure 6). A motorized synovial resector is used to smooth the degenerative parts or to remove the dissected parts of the meniscus. Standard arthroscopic instruments like a probe, holding forceps, or dissecting scissors are also useful. For optional capsular release, an arthroscopic knife is utilized. Given that repair of the meniscus has to always be taken into account, instruments that are adequate for a meniscal suture should always be available.

\section{Surgical procedure}

The arthroscope is advanced through the ALP in $90^{\circ}$ flexion, pointing in the direction of the intercondylar notch in order to prevent any harm to the articular cartilage. The 


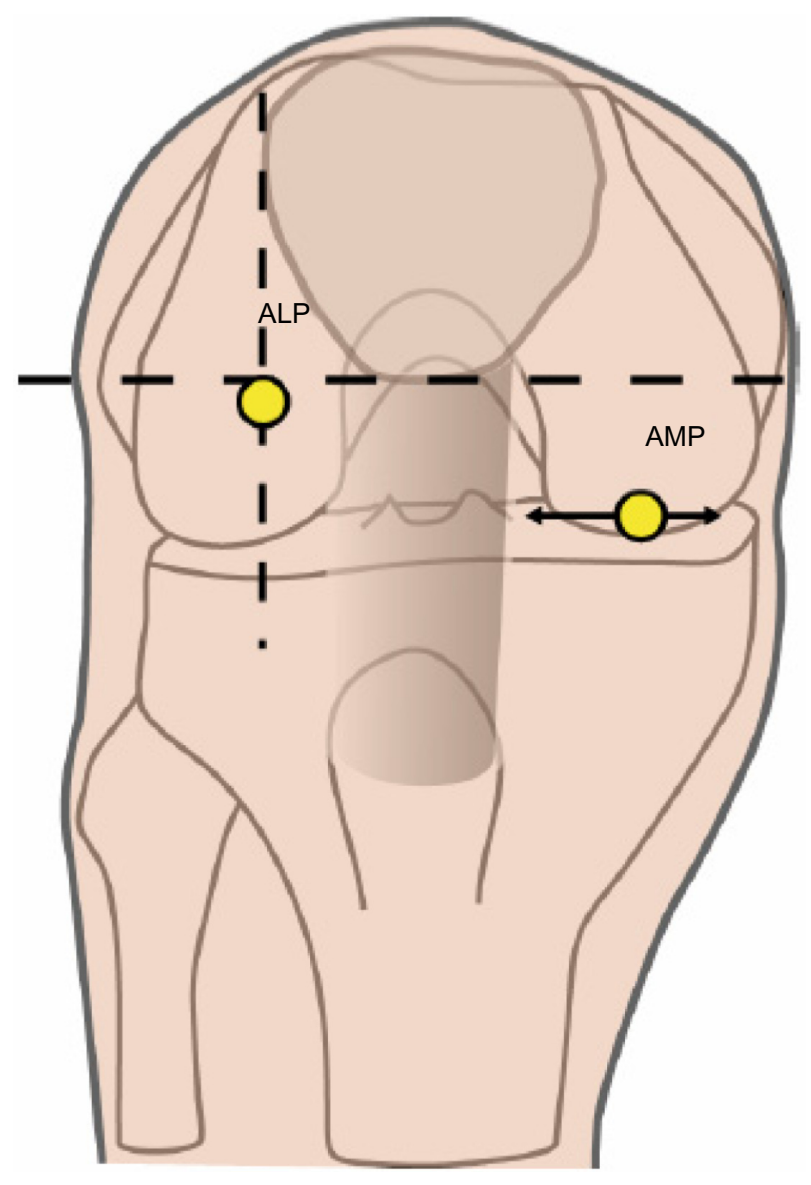

Figure 5 Illustration of the position of the ALP and AMP. Abbreviations: ALP, anterolateral portal; AMP, anteromedial portal.

knee is extended and the diagnostic arthroscopy begins in the superior recess of the knee joint. The lateral and medial recesses are visualized, and loose bodies are ruled out in these locations. The cartilage of the patellofemoral joint is assessed. The scope is then pulled back slightly and the medial tibiofemoral joint space is evaluated. In some cases, some degree of flexion improves the visibility of the posterior part of the knee joint. If the medial joint space is very narrow, the posterior oblique ligament (POL) is released in order to generate more working space and to obtain a better overview of the posterior meniscus. First, an AMP is established, as described. Then, the POL can be released either intrarticularly

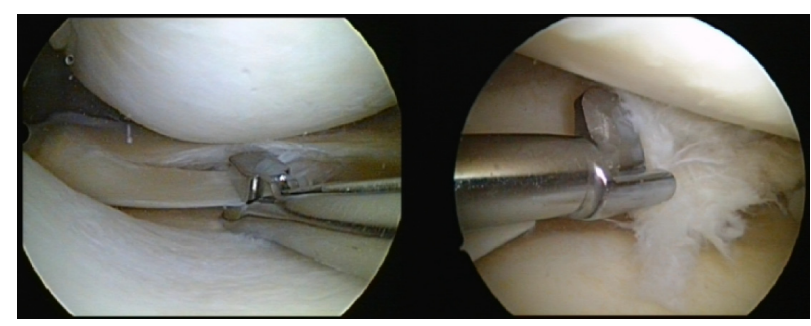

Figure 6 Straight and lateral punch forceps instruments. by puncturing the posteromedial capsule above the medial meniscus with an arthroscopic knife, or by using a spinal needle extraarticularly. While putting valgus stress on the knee, the joint space will open about $5 \mathrm{~mm}$. The cartilage and the meniscus are evaluated with a probe.

In the intercondylar notch, the tension of the ACL is evaluated in $90^{\circ}$ of flexion. Due to the synovial coverage, the posterior cruciate ligament cannot be assessed efficiently. The lateral joint is evaluated in a "figure-of-four" position of the knee $\left(90^{\circ}\right.$ flexion, varus stress, and external hip rotation). For better visualization of the anterior horn of the lateral meniscus, the arthroscope may be switched to the AMP, if necessary. Once again, the cartilage and meniscus are probed. After video documentation of all arthroscopic findings, the decision has to be made regarding whether the meniscus should be repaired or resected.

For flap tears, resection begins at the stable part of the lesion until the whole fragment is dissected. Alternatively, the flap can be aspirated using suction, and it may be dissected using the shaving instrument.

With horizontal tears, manual investigation with a probe identifies the unstable layer. In most cases, the inferior part of the lesion is the unstable one. The unstable layer is resected with the punch forceps, beginning at the most posterior part until the remaining rim seems to be stable. The layer is resected piece by piece, and the fragments are removed by a suction device or a motorized shaver. Vertical tears can be resected like horizontal tears, or cut at the endpoints of the tear and removed in one piece. In complex degenerative tears, it is often easier to resect the worn parts and fragments with the shaver first in order to improve visualization. Then, the main fragments can be better identified and treated.

\section{One step beyond meniscal resection}

The natural progression of problems with the knee after a meniscectomy is the development of OA. Therefore, research is being conducted to prevent arthritis by replacing the resected meniscus. Several attempts to accomplish this have been made using autografts derived from the fat pad, tendon, cartilage, periosteum, synovial flap, and perichondrium. Most of these procedures did not yield satisfactory results. ${ }^{58}$ In 1989, Milachowski et al ${ }^{59}$ reported the first attempt at meniscal allograft transplantation in humans. Due to improvements in surgical techniques, patient selection, graft processing, and preparation, the meniscal allograft transplantation has shown promising short- and mid-term results. ${ }^{60,61} \mathrm{In}$ contrast to allogenic transplantation, the collagen meniscus implant (CMI ${ }^{\mathrm{TM}}$, Ivy Sports Medicine, Germany) is intended 


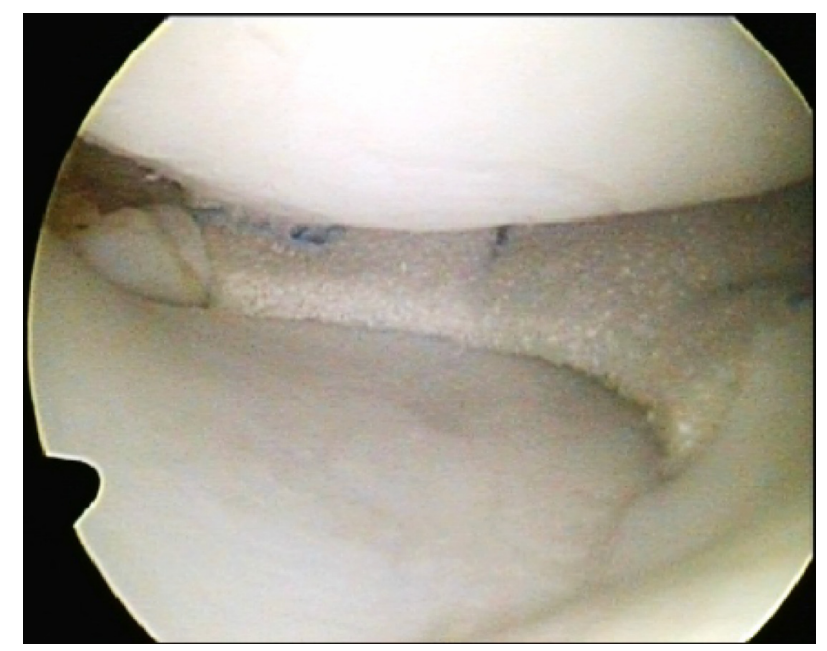

Figure 7 Actifit $^{\circledR}$ (Orteq ${ }^{\circledR}$ Ltd, London, UK) implant for partial medial meniscus reconstruction.

to fill larger defects following partial resection. The implant is made from type 1 collagen fibers from purified bovine Achilles tendon, which is supplemented with glycosaminoglycans. The CMI ${ }^{\mathrm{TM}}$ needs an intact rim so it can be attached. According to a multicenter study by Rodkey et al, ${ }^{62}$ only patients who underwent prior meniscal surgical procedures improved significantly after treatment with the CMITM Recently, a synthetic scaffold (Actifit ${ }^{\circledR}$, Orteq ${ }^{\circledR}$ Ltd, London, UK) consisting of polyurethane polymers and biodegradable polyester segments was developed. This implant allows for an ingrowth of tissue to build up new meniscal tissue. ${ }^{63}$ Even though indications for this scaffold are the same as for the $\mathrm{CMI}^{\mathrm{TM}}$, the Actifit $^{\circledR}$ (Figure 7) is more robust and therefore easier to implant. Verdonk et al ${ }^{64}$ showed tissue ingrowth after 3 months in over $80 \%$ of their patients. In a multicenter, single-arm, proof-of-principle study, significant improvements in patients' clinical scores were observed after 2 years, while nine out of 52 patients (17.3\%) required reoperation due to treatment failure. ${ }^{65}$

\section{Rehabilitation}

There is no consensus on which treatment option is the best for patients following meniscectomy and meniscus repair. Physical therapy consisting of home exercises seems to be effective in improving patient-reported knee function and range of motion in partial meniscectomy ${ }^{66}$ In our practice, patients should walk on crutches with partial weight-bearing until effusion and tenderness of the joint disappear. Two weeks postoperatively, patients may begin with muscle strengthening and proprioceptive training. Normally, the rehabilitation ends after 4 weeks.
In contrast, the rehabilitation for meniscal repairs or replacements is much more extensive and is based on the biomechanical function of both menisci. There is no weight-bearing for the first 6 weeks, besides complete axial loading in a full-extension brace in medial meniscus repairs. Range of motion is limited to $60^{\circ}$ and $90^{\circ}$ of flexion for 6 weeks for lateral and medial meniscus repairs, respectively. After the first 6 weeks, weight-bearing and range of motion are increased according to the functional status of the knee.

\section{Clinical outcomes}

Recently, several studies and meta-analyses compared the clinical outcomes between meniscal repairs and partial resections. Stein et $\mathrm{al}^{67}$ found a significant benefit for clinical symptoms and sports activity in the long-term follow up of 8.8 years in 46 patients with meniscal repair compared to partial resection. Petty and Lubowitz ${ }^{68}$ pooled the data of five studies that conducted a minimum follow up of 8 years. Significant radiographic signs of OA were found after partial meniscectomy, but the clinical symptoms of knee OA were not significant. In contrast, a meta-analysis by Nepple et a ${ }^{69}$ compiled from 13 studies reporting the outcomes of meniscal repairs with greater than 5 years of follow up showed a failure rate between $22.3 \%$ and $24.3 \%$. Paxton et al $^{70}$ summarized these findings in their review, and concluded that meniscal repairs have a higher reoperation rate than partial meniscectomies, and are associated with better long-term outcomes.

\section{Conclusion}

As Vidal ${ }^{71}$ stated, more orthopedic surgeons should join the "Save the Meniscus" society, since the menisci are not "appendices" of the knee, and given that our default responsibility as surgeons should always be to repair first and resect only when repair is not feasible. Repair devices have become easier to use, even though anatomic knowledge of the surrounding nerves and vessels of the knee is still necessary so as to avoid serious complications. Meniscal replacement or transplantation are highly demanding surgical procedures that might be good solutions in highly selected patients. Not much is known about biologic healing enhancers like fibrin clots or platelet-rich plasma, but further investigations might lead to a significant decrease of retear rates following meniscal repairs or replacements.

\section{Disclosure}

The authors report no conflicts of interest in this work. 


\section{References}

1. van Saase JL, van Romunde LK, Cats A, Vandenbroucke JP, Valkenburg HA. Epidemiology of osteoarthritis: Zoetermeer survey. Comparison of radiological osteoarthritis in a Dutch population with that in 10 other populations. Ann Rheum Dis. 1989;48(4): $271-280$.

2. Losina E, Weinstein AM, Reichmann WM, et al. Lifetime risk and age at diagnosis of symptomatic knee osteoarthritis in the US. Arthritis Care Res (Hoboken). 2013;65(5):703-711.

3. Fairbank TJ. Knee joint changes after meniscectomy. J Bone Joint Surg Br. 1948;30B(4):664-670.

4. Allen PR, Denham RA, Swan AV. Late degenerative changes after meniscectomy. Factors affecting the knee after operation. J Bone Joint Surg Br. 1984;66(5):666-671.

5. Jørgensen U, Sonne-Holm S, Lauridsen F, Rosenklint A. Long-term follow-up of meniscectomy in athletes. A prospective longitudinal study. J Bone Joint Surg Br. 1987;69(1):80-83.

6. Englund M, Lohmander LS. Risk factors for symptomatic knee osteoarthritis fifteen to twenty-two years after meniscectomy. Arthritis Rheum. 2004;50(9):2811-2819.

7. Papalia R, Del Buono A, Osti L, Denaro V, Maffulli N. Meniscectomy as a risk factor for knee osteoarthritis: a systematic review. Br Med Bull. 2011;99:89-106.

8. Pengas IP, Assiotis A, Nash W, Hatcher J, Banks J, McNicholas MJ. Total meniscectomy in adolescents: a 40-year follow-up. J Bone Joint Surg Br. 2012;94(12):1649-1654.

9. Englund M, Guermazi A, Roemer FW, et al. Meniscal tear in knees without surgery and the development of radiographic osteoarthritis among middle-aged and elderly persons: The Multicenter Osteoarthritis Study. Arthritis Rheum. 2009;60(3):831-839.

10. Katz JN, Martin SD. Meniscus - friend or foe: epidemiologic observations and surgical implications. Arthritis Rheum. 2009;60(3):633-635.

11. Setton LA, Zhu W, Mow VC. The biphasic poroviscoelastic behavior of articular cartilage: role of the surface zone in governing the compressive behavior. J Biomech. 1993;26(4-5):581-592.

12. Ateshian GA, Lai WM, Zhu WB, Mow VC. An asymptotic solution for the contact of two biphasic cartilage layers. J Biomech. 1994;27(11):1347-1360.

13. Ahmed AM, Burke DL. In-vitro measurement of static pressure distribution in synovial joints - Part I: Tibial surface of the knee. J Biomech Eng. 1983;105(3):216-225.

14. Ihn JC, Kim SJ, Park IH. In vitro study of contact area and pressure distribution in the human knee after partial and total meniscectomy. Int Orthop. 1993;17(4):90-92.

15. Kummer B. Biomechanik des Meniskus. [Biomechanics of the meniscus]. Orthopäde. 1994;23(2):90-102. German.

16. Allaire R, Muriuki M, Gilbertson L, Harner CD. Biomechanical consequences of a tear of the posterior root of the medial meniscus. Similar to total meniscectomy. J Bone Joint Surg Am. 2008;90(9): 1922-1931.

17. Marzo JM, Gurske-DePerio J. Effects of medial meniscus posterior horn avulsion and repair on tibiofemoral contact area and peak contact pressure with clinical implications. Am J Sports Med. 2009;37(1):124-129.

18. Paletta GA, Manning T, Snell E, Parker R, Bergfeld J. The effect of allograft meniscal replacement on intraarticular contact area and pressures in the human knee. A biomechanical study. Am J Sports Med. 1997;25(5):692-698.

19. Shoemaker SC, Markolf KL. The role of the meniscus in the anterior-posterior stability of the loaded anterior cruciate-deficient knee. Effects of partial versus total excision. J Bone Joint Surg Am. 1986;68(1):71-79.

20. Petersen W, Tillmann B. Collagenous fibril texture of the human knee joint menisci. Anat Embryol (Berl). 1998;197(4):317-324.

21. Kohn D, Moreno B. Meniscus insertion anatomy as a basis for meniscus replacement: a morphological cadaveric study. Arthroscopy. 1995;11(1):96-103.
22. Thompson WO, Thaete FL, Fu FH, Dye SF. Tibial meniscal dynamics using three-dimensional reconstruction of magnetic resonance images. Am J Sports Med. 1991;19(3):210-215; discussion 215-216.

23. Vedi V, Williams A, Tennant SJ, Spouse E, Hunt DM, Gedroyc WM. Meniscal movement. An in-vivo study using dynamic MRI. J Bone Joint Surg Br. 1999;81(1):37-41.

24. Arnoczky SP, Warren RF. Microvasculature of the human meniscus. Am J Sports Med. 1982;10(2):90-95.

25. Zimny ML, Albright DJ, Dabezies E. Mechanoreceptors in the human medial meniscus. Acta Anat (Basel). 1988;133(1):35-40.

26. Noble J, Hamblen DL. The pathology of the degenerate meniscus lesion. J Bone Joint Surg Br. 1975;57(2):180-186.

27. Poehling GG, Ruch DS, Chabon SJ. The landscape of meniscal injuries. Clin Sports Med. 1990;9(3):539-549.

28. Hede A, Jensen DB, Blyme P, Sonne-Holm S. Epidemiology of meniscal lesions in the knee. 1,215 open operations in Copenhagen 1982-1984. Acta Orthop Scand. 1990;61(5):435-437.

29. Steinbrück K. [Epidemiology of sports injuries - 25-year-analysis of sports orthopedic-traumatologic ambulatory care]. Sportverletz Sportschaden. 1999;13(2):38-52. German.

30. Clayton RA, Court-Brown CM. The epidemiology of musculoskeletal tendinous and ligamentous injuries. Injury. 2008;39(12): 1338-1344.

31. Lind M, Menhert F, Pedersen AB. The first results from the Danish ACL reconstruction registry: epidemiologic and 2 year follow-up results from 5,818 knee ligament reconstructions. Knee Surg Sports Traumatol Arthrosc. 2009;17(2):117-124.

32. Henning CE, Clark JR, Lynch MA, Stallbaumer R, Yearout KM, Vequist SW. Arthroscopic meniscus repair with a posterior incision. Instr Course Lect. 1988;37:209-221.

33. Shelbourne KD, Heinrich J. The long-term evaluation of lateral meniscus tears left in situ at the time of anterior cruciate ligament reconstruction. Arthroscopy. 2004;20(4):346-351.

34. Shelbourne KD, Roberson TA, Gray T. Long-term evaluation of posterior lateral meniscus root tears left in situ at the time of anterior cruciate ligament reconstruction. Am J Sports Med. 2011;39(7):1439-1443.

35. Terry GC, Tagert BE, Young MJ. Reliability of the clinical assessment in predicting the cause of internal derangements of the knee. Arthroscopy. 1995;11(5):568-576.

36. Mohan BR, Gosal HS. Reliability of clinical diagnosis in meniscal tears. Int Orthop. 2007;31(1):57-60.

37. Ercin E, Kaya I, Sungur I, Demirbas E, Ugras AA, Cetinus EM. History, clinical findings, magnetic resonance imaging, and arthroscopic correlation in meniscal lesions. Knee Surg Sports Traumatol Arthrosc. 2012;20(5):851-856.

38. Dervin GF, Stiell IG, Wells GA, Rody K, Grabowski J. Physicians' accuracy and interrator reliability for the diagnosis of unstable meniscal tears in patients having osteoarthritis of the knee. Can J Surg. 2001;44(4):267-274.

39. Crawford R, Walley G, Bridgman S, Maffulli N. Magnetic resonance imaging versus arthroscopy in the diagnosis of knee pathology, concentrating on meniscal lesions and ACL tears: a systematic review. $\mathrm{Br}$ Med Bull. 2007;84:5-23.

40. Subhas N, Sakamoto FA, Mariscalco MW, Polster JM, Obuchowski NA, Jones MH. Accuracy of MRI in the diagnosis of meniscal tears in older patients. AJR Am J Roentgenol. 2012;198(6):W575-W580.

41. Konan S, Rayan F, Haddad FS. Do physical diagnostic tests accurately detect meniscal tears? Knee Surg Sports Traumatol Arthrosc. 2009;17(7):806-811.

42. Noble J. Lesions of the menisci. Autopsy incidence in adults less than fifty-five years old. J Bone Joint Surg Am. 1977;59(4):480-483.

43. Lee CH, Song IS, Jang SW, Cha HE. Results of arthroscopic partial meniscectomy for lateral discoid meniscus tears associated with new technique. Knee Surg Relat Res. 2013;25(1):30-35.

44. Ozkoc G, Circi E, Gonc U, Irgit K, Pourbagher A, Tandogan RN. Radial tears in the root of the posterior horn of the medial meniscus. Knee Surg Sports Traumatol Arthrosc. 2008;16(9):849-854. 
45. Seo HS, Lee SC, Jung KA. Second-look arthroscopic findings after repairs of posterior root tears of the medial meniscus. Am J Sports Med. 2011;39(1):99-107.

46. Bloome DM, Blevins FT, Paletta GA, Newcomer JK, Cashmore B, Turker R. Meniscal repair in very young children. Arthroscopy. 2000;16(5):545-549.

47. Kraus T, Heidari N, Švehlík M, Schneider F, Sperl M, Linhart W. Outcome of repaired unstable meniscal tears in children and adolescents. Acta Orthop. 2012;83(3):261-266.

48. Longo UG, Campi S, Romeo G, Spiezia F, Maffulli N, Denaro V. Biological strategies to enhance healing of the avascular area of the meniscus. Stem Cells Int. 2012;2012:528359.

49. Scordino LE, Deberardino TM. Biologic enhancement of meniscus repair. Clin Sports Med. 2012;31(1):91-100.

50. Cooper DE, Arnoczky SP, Warren RF. Meniscal repair. Clin Sports Med. 1991;10(3):529-548.

51. Laible C, Stein DA, Kiridly DN. Meniscal repair. J Am Acad Orthop Surg. 2013;21(4):204-213

52. Cannon WD, Vittori JM. The incidence of healing in arthroscopic meniscal repairs in anterior cruciate ligament-reconstructed knees versus stable knees. Am J Sports Med. 1992;20(2):176-181.

53. Tenuta JJ, Arciero RA. Arthroscopic evaluation of meniscal repairs Factors that effect healing. Am J Sports Med. 1994;22(6):797-802.

54. Kimura M, Shirakura K, Hasegawa A, Kobuna Y, Niijima M. Second look arthroscopy after meniscal repair. Factors affecting the healing rate. Clin Orthop Relat Res. 1995;(314):185-191.

55. Barrett GR, Field MH, Treacy SH, Ruff CG. Clinical results of meniscus repair in patients 40 years and older. Arthroscopy. 14(8):824-829.

56. Boyd KT, Myers PT. Meniscus preservation; rationale, repair techniques and results. Knee. 2003;10(1):1-11.

57. Gwathmey FW, Golish SR, Diduch DR. Complications in brief: meniscus repair. Clin Orthop Relat Res. 2012;470(7):2059-2066.

58. Liu C, Toma IC, Mastrogiacomo M, Krettek C, von Lewinski G, Jagodzinski M. Meniscus reconstruction: today's achievements and premises for the future. Arch Orthop Trauma Surg. 2013;133(1): 95-109.

59. Milachowski KA, Weismeier K, Wirth CJ. Homologous meniscus transplantation. Experimental and clinical results. Int Orthop. 1989;13(1):1-11.

60. Lubowitz JH, Verdonk PC, Reid JB 3rd, Verdonk R. Meniscus allograft transplantation: a current concepts review. Knee Surg Sports Traumatol Arthrosc. 2007;15(5):476-492.
61. Verdonk PC, Demurie A, Almqvist KF, Veys EM, Verbruggen G, Verdonk R. Transplantation of viable meniscal allograft. Survivorship analysis and clinical outcome of one hundred cases. J Bone Joint Surg Am. 2005;87(4):715-724.

62. Rodkey WG, DeHaven KE, Montgomery WH, et al. Comparison of the collagen meniscus implant with partial meniscectomy. A prospective randomized trial. J Bone Joint Surg Am. 2008;90(7):1413-1426.

63. Vrancken AC, Buma P, van Tienen TG. Synthetic meniscus replacement: a review. Int Orthop. 2013;37(2):291-299.

64. Verdonk R, Verdonk P, Huysse W, Forsyth R, Heinrichs EL. Tissue ingrowth after implantation of a novel, biodegradable polyurethane scaffold for treatment of partial meniscal lesions. Am J Sports Med. 2011;39(4):774-782.

65. Verdonk P, Beaufils P, Bellemans J, et al; Actfit Study Group. Successful treatment of painful irreparable partial meniscal defects with a polyurethane scaffold: two-year safety and clinical outcomes. Am J Sports Med. 2012;40(4):844-853.

66. Dias JM, Mazuquin BF, Mostagi FQ, et al. The effectiveness of postoperative physical therapy treatment in patients who have undergone arthroscopic partial meniscectomy: systematic review with metaanalysis. J Orthop Sports Phys Ther. 2013;43(8):560-576.

67. Stein T, Mehling AP, Welsch F, von Eisenhart-Rothe R, Jäger A. Long-term outcome after arthroscopic meniscal repair versus arthroscopic partial meniscectomy for traumatic meniscal tears. Am J Sports Med. 2010;38(8):1542-1548.

68. Petty CA, Lubowitz JH. Does arthroscopic partial meniscectomy result in knee osteoarthritis? A systematic review with a minimum of 8 years' follow-up. Arthroscopy. 2011;27(3):419-424.

69. Nepple JJ, Dunn WR, Wright RW. Meniscal repair outcomes at greater than five years: a systematic literature review and meta-analysis. J Bone Joint Surg Am. 2012;94(24):2222-2227.

70. Paxton ES, Stock MV, Brophy RH. Meniscal repair versus partial meniscectomy: a systematic review comparing reoperation rates and clinical outcomes. Arthroscopy. 2011;27(9):1275-1288.

71. Vidal AF. The save the meniscus society: commentary on an article by Jeffrey J. Nepple MD, et al: "Meniscal repair outcomes at greater than five years. A systematic literature review and meta-analysis". J Bone Joint Surg Am. 2012;94(24):e186.
Orthopedic Research and Reviews

\section{Publish your work in this journal}

Orthopedic Research and Reviews is an international, peer-reviewed, open access journal focusing on the patho-physiology of the musculoskeletal system, trauma, surgery and other corrective interventions to restore mobility and function. Advances in new technologies, materials, techniques and pharmacological agents are particularly welcome. The journal welcomes

\section{Dovepress}

original research, clinical studies, reviews \& evaluations, expert opinion and commentary, case reports and extended reports. The manuscript management system is completely online and includes a very quick and fair peer-review system, which is all easy to use. Visit http://www.dovepress. com/testimonials.php to read real quotes from published authors. 